\title{
Pfaffianization of the q-difference version of the two-dimensional Toda lattice equation
}

Gegenhasi ${ }^{a, b}$, Xing-Biao HU ${ }^{a}$, and Hon-Wah Tam $^{c}$

${ }^{a}$ Institute of Computational Mathematics and Scientific Engineering Computing, AMSS, Chinese Academy of Sciences, P.O. Box 2719, Beijing 100080, P.R. CHINA

${ }^{b}$ Graduate School of the Chinese Academy of Sciences, Beijing, P.R. CHINA

E-mail: gegen@mail.amss.ac.cn,hxb@lsec.cc.ac.cn

${ }^{c}$ Department of Computer Science, Hong Kong Baptist University Kowloon Tong, Hong Kong, P.R. CHINA

E-mail:tam@comp.hkbu.edu.hk

This article is a part of the special issue titled "Symmetries and Integrability of Difference Equations (SIDE VI)"

\begin{abstract}
We apply the pfaffianization procedure to the q-difference version of the two-dimensional Toda lattice equation to produce the pfaffian analogue of the q-difference twodimensional Toda lattice equation. A solution to the pfaffianized system is expressed in terms of the q-exponential functions.
\end{abstract}

\section{Introduction}

The method of pfaffianization developed by Hirota and Ohta in 1991 has provided us with a powerful tool to produce coupled systems for several continuous $(2+1)$-dimensional soliton equations such as the KP and DS equations etc. [1, 2, 3]. Very recently, such a pfaffianization procedure has been further extended to the differential-difference case and fully discrete case $[4,5,6]$. It is known that in the literature there exists much research on the so-called $q$-difference equations. An active area of particular interest is $q$-difference versions of integrable equations. Therefore, it is quite natural to see if the method of pfaffianization may be generalized to the $q$-difference case of integrable equations. The purpose of this paper is to apply the pfaffianization procedure to the q-difference version for the two-dimensional Toda lattice equation. As a result, a coupled system for the q-difference version of the two-dimensional Toda lattice equation is derived.

\section{Pfaffianization of the q-difference version for the 2DTL}

In this section, we devote to Pfaffianizing the q-difference version of two-dimensional Toda lattice equation [7],

$$
\begin{aligned}
& \tau_{n}(x, y)\left(\delta_{q_{1}^{\alpha}, x} \delta_{q_{2}^{\beta}, y}\right) \tau_{n}(x, y)-\delta_{q_{1}^{\alpha}, x} \tau_{n}(x, y) \delta_{q_{2}^{\beta}, y} \tau_{n}(x, y) \\
& =\tau_{n+1}\left(x, q_{2}^{\beta} y\right) \tau_{n-1}\left(q_{1}^{\alpha} x, y\right)-\tau_{n}\left(q_{1}^{\alpha} x, q_{2}^{\beta} y\right) \tau_{n}(x, y)
\end{aligned}
$$


where for an arbitrary function $F(x)$, the q-difference operator $\delta_{q^{\alpha}, x}$ is defined by

$$
\delta_{q^{\alpha}, x} F(x)=\frac{F(x)-F\left(q^{\alpha} x\right)}{(1-q) x} .
$$

We can write equation (2.1) in its equivalent form,

$$
\begin{aligned}
& \tau_{n}\left(q_{1}^{\alpha} x, q_{2}^{\beta} y\right) \tau_{n}(x, y)-\tau_{n}\left(q_{1}^{\alpha} x, y\right) \tau_{n}\left(x, q_{2}^{\beta} y\right)= \\
& \quad\left(1-q_{1}\right)\left(1-q_{2}\right) x y\left(\tau_{n+1}\left(x, q_{2}^{\beta} y\right) \tau_{n-1}\left(q_{1}^{\alpha} x, y\right)-\tau_{n}\left(q_{1}^{\alpha} x, q_{2}^{\beta} y\right) \tau_{n}(x, y)\right) .
\end{aligned}
$$

In [7], the solution of the equation (2.1) is given by the following $N \times N$ Wronskian (Casorati) determinant:

$$
\tau_{n}(x, y)=\left|\begin{array}{cccc}
f_{1}^{(n)}(x, y) & f_{1}^{(n+1)}(x, y) & \cdots & f_{1}^{(n+N-1)}(x, y) \\
f_{2}^{(n)}(x, y) & f_{2}^{(n+1)}(x, y) & \cdots & f_{2}^{(n+N-1)}(x, y) \\
\vdots & \vdots & & \vdots \\
f_{N}^{(n)}(x, y) & f_{N}^{(n+1)}(x, y) & \cdots & f_{N}^{(n+N-1)}(x, y)
\end{array}\right|,
$$

where $f_{k}^{(n)}(x, y), k=1,2, \cdots, N$ satisfy the following dispersion relations:

$$
\begin{aligned}
& \delta_{q_{1}^{\alpha}, x} f_{k}^{(n)}(x, y)=-f_{k}^{(n+1)}(x, y), \\
& \delta_{q_{2}^{\beta}, y} f_{k}^{(n)}(x, y)=f_{k}^{(n-1)}(x, y) .
\end{aligned}
$$

In order to pfaffianize the q-difference version of 2DTL, we require pfaffians with entries satisfying the pfaffianized form of the dispersion relation (2.4-2.5), i.e. we choose pfaffians whose entries satisfy:

$$
\begin{gathered}
\operatorname{pf}(i, j)\left(q_{1}^{\alpha} x, y\right)=\operatorname{pf}(i, j)+\left(1-q_{1}\right) x \operatorname{pf}(i+1, j)+\left(1-q_{1}\right) x \operatorname{pf}(i, j+1) \\
+\left(1-q_{1}\right)^{2} x^{2} \operatorname{pf}(i+1, j+1), \\
\operatorname{pf}(i, j)\left(x, q_{2}^{\beta} y\right)=\operatorname{pf}(i, j)-\left(1-q_{2}\right) y \operatorname{pf}(i-1, j)-\left(1-q_{2}\right) y \operatorname{pf}(i, j-1) \\
+\left(1-q_{2}\right)^{2} y^{2} \operatorname{pf}(i-1, j-1),
\end{gathered}
$$

where we have denoted $\operatorname{pf}(i, j)_{n}(x, y)$ to be $\operatorname{pf}(i, j)$ for simplicity without any confusion.

For even value of $N$, if we take

$$
\tau_{n}(x, y)=\operatorname{pf}(1,2,3, \cdots, N)
$$

together with $\operatorname{pf}(i, c)=\left[-\left(1-q_{1}\right) x\right]^{N+1-i}, \operatorname{pf}(i, d)=\left[\left(1-q_{2}\right) y\right]^{i}$ and $\operatorname{pf}(c, d)=0$, then we 
can obtain the following formulae for $\tau_{n}(x, y)$ by using the relations(2.6-2.8):

$$
\begin{aligned}
& \tau_{n}\left(q_{1}^{\alpha} x, y\right)=\operatorname{pf}(1, \cdots, N, N+1, c), \\
& \tau_{n}\left(x, q_{2}^{\beta} y\right)=\operatorname{pf}(0,1, \cdots, N, d), \\
& \tau_{n}\left(q_{1}^{\alpha} x, q_{2}^{\beta} y\right)=\frac{1}{1+\left(1-q_{1}\right)\left(1-q_{2}\right) x y} \operatorname{pf}(0,1, \cdots, N, N+1, c, d), \\
& \tau_{n-1}\left(q_{1}^{\alpha} x, y\right)=-\frac{1}{\left(1-q_{1}\right) x} \operatorname{pf}(0,1, \cdots, N, c), \\
& \tau_{n+1}\left(x, q_{2}^{\beta} y\right)=\frac{1}{\left(1-q_{2}\right) y} \operatorname{pf}(1, \cdots, N, N+1, d), \\
& \tau_{n+1}\left(q_{1}^{\alpha} x, q_{2}^{\beta} y\right)=-\frac{\left(1-q_{1}\right) x}{\left(1-q_{2}\right) y\left[1+\left(1-q_{1}\right)\left(1-q_{2}\right) x y\right]} \\
& \operatorname{pf}(1,2, \cdots, N+1, N+2, c, d) .
\end{aligned}
$$

Following Hirota and Ohta's method, we now review two basic pfaffian identities [8]:

$$
\begin{aligned}
& \operatorname{pf}\left(\alpha, \beta, \gamma, \delta, p_{1}, p_{2}, \cdots, p_{2 n}\right) \operatorname{pf}\left(p_{1}, p_{2}, \cdots, p_{2 n}\right) \\
& \quad-\operatorname{pf}\left(\alpha, \beta, p_{1}, p_{2}, \cdots, p_{2 n}\right) \operatorname{pf}\left(\gamma, \delta, p_{1}, p_{2}, \cdots, p_{2 n}\right) \\
& \quad+\operatorname{pf}\left(\alpha, \gamma, p_{1}, p_{2}, \cdots, p_{2 n}\right) \operatorname{pf}\left(\beta, \delta, p_{1}, p_{2}, \cdots, p_{2 n}\right) \\
& \quad-\operatorname{pf}\left(\alpha, \delta, p_{1}, p_{2}, \cdots, p_{2 n}\right) \operatorname{pf}\left(\beta, \gamma, p_{1}, p_{2}, \cdots, p_{2 n}\right)=0
\end{aligned}
$$

and

$$
\begin{aligned}
& \operatorname{pf}\left(\alpha, \beta, \gamma, p_{1}, p_{2}, \cdots, p_{2 n-1}\right) \operatorname{pf}\left(\delta, p_{1}, p_{2}, \cdots, p_{2 n-1}\right) \\
& \quad-\operatorname{pf}\left(\alpha, \beta, \delta, p_{1}, p_{2}, \cdots, p_{2 n-1}\right) \operatorname{pf}\left(\gamma, p_{1}, p_{2}, \cdots, p_{2 n-1}\right) \\
& \quad+\operatorname{pf}\left(\alpha, \gamma, \delta, p_{1}, p_{2}, \cdots, p_{2 n-1}\right) \operatorname{pf}\left(\beta, p_{1}, p_{2}, \cdots, p_{2 n-1}\right) \\
& \quad-\operatorname{pf}\left(\beta, \gamma, \delta, p_{1}, p_{2}, \cdots, p_{2 n-1}\right) \operatorname{pf}\left(\alpha, p_{1}, p_{2}, \cdots, p_{2 n-1}\right)=0 .
\end{aligned}
$$

In the case of q-difference version of 2DTL equation, we require the following identities

$$
\begin{aligned}
& \operatorname{pf}(0, N+1, c, d, 1, \cdots, N) \operatorname{pf}(1, \cdots, N) \\
& -\operatorname{pf}(N+1, c, 1,, \cdots, N) \operatorname{pf}(0, d, 1, \cdots, N) \\
& \quad+\operatorname{pf}(N+1, d, 1, \cdots, N) \operatorname{pf}(0, c, 1, \cdots, N) \\
& \quad-\operatorname{pf}(0, N+1,1, \cdots, N) \operatorname{pf}(c, d, 1, \cdots, N)=0,
\end{aligned}
$$

obtained from (2.16). This leads to the equation

$$
\begin{aligned}
{[1+} & \left.\left(1-q_{1}\right)\left(1-q_{2}\right) x y\right] \tau_{n}\left(q_{1}^{\alpha} x, q_{2}^{\beta} y\right) \tau_{n}(x, y)-\tau_{n}\left(q_{1}^{\alpha} x, y\right) \tau_{n}\left(x, q_{2}^{\beta} y\right) \\
& -\left(1-q_{1}\right)\left(1-q_{2}\right) x y \tau_{n+1}\left(x, q_{2}^{\beta} y\right) \tau_{n-1}\left(q_{1}^{\alpha} x, y\right) \\
& +\left(1-q_{1}\right)\left(1-q_{2}\right) x y\left[1+\left(1-q_{1}\right)\left(1-q_{2}\right) x y\right] \tilde{\sigma}_{n}\left(q_{1}^{\alpha} x, q_{2}^{\beta} y\right) \sigma_{n}(x, y)=0 .
\end{aligned}
$$

Here the new fields $\tilde{\sigma}_{n}$ and $\sigma_{n}$ are also Pfaffians of the form

$$
\tilde{\sigma}_{n}=\operatorname{pf}(2,3, \cdots, N-1), \quad \sigma_{n}=\operatorname{pf}(0,1,2, \cdots, N, N+1) .
$$


For the Pfaffians $\tilde{\sigma}_{n}$ and $\sigma_{n}$, we can derive the following formulae by employing equations (2.6-2.8):

$$
\begin{aligned}
& \tilde{\sigma}_{n}\left(q_{1}^{\alpha} x, y\right)=-\frac{1}{\left(1-q_{1}\right) x} \operatorname{pf}(2,3, \cdots, N-1, N, c), \\
& \tilde{\sigma}_{n}\left(x, q_{2}^{\beta} y\right)=\frac{1}{\left(1-q_{2}\right) y} \operatorname{pf}(1,2, \cdots, N-1, d), \\
& \tilde{\sigma}_{n}\left(q_{1}^{\alpha} x, q_{2}^{\beta} y\right)=-\frac{1}{\left(1-q_{1}\right)\left(1-q_{2}\right) x y\left[1+\left(1-q_{1}\right)\left(1-q_{2}\right) x y\right]} \\
& \operatorname{pf}(1,2, \cdots, N-1, N, c, d), \\
& \tilde{\sigma}_{n-1}\left(q_{1}^{\alpha} x, y\right)=\frac{1}{\left(1-q_{1}\right)^{2} x^{2}} \operatorname{pf}(1,2, \cdots, N-1, c), \\
& \tilde{\sigma}_{n+1}\left(x, q_{2}^{\beta} y\right)=\frac{1}{\left(1-q_{2}\right)^{2} y^{2}} \operatorname{pf}(2,3, \cdots, N-1, N, d), \\
& \tilde{\sigma}_{n+1}\left(q_{1}^{\alpha} x, q_{2}^{\beta} y\right)=\frac{1}{\left(1-q_{2}\right)^{2} y^{2}\left[1+\left(1-q_{1}\right)\left(1-q_{2}\right) x y\right]} \\
& \sigma_{n}\left(q_{1}^{\alpha} x, y\right)=-\left[\left(1-q_{1}\right) x\right] \operatorname{pf}(0,1, \cdots, N+1, N+2, c), \\
& \sigma_{n}\left(x, q_{2}^{\beta} y\right)=\left(1-q_{2}\right) y \operatorname{pf}(-1,0, \cdots, N, N+1, c, d), \\
& \sigma_{n-1}\left(q_{1}^{\alpha} x, y\right)=\operatorname{pf}(-1,0, \cdots, N, N+1, c), \\
& \sigma_{n+1}\left(x, q_{2}^{\beta} y\right)=\operatorname{pf}(0,1, \cdots, N+1, N+2, d) .
\end{aligned}
$$

The introduction of two new fields $\tilde{\sigma}_{n}$ and $\sigma_{n}$ means that our system is no longer closed. Thus we need to look for further identities. With the help of the Pfaffian identity (2.17), we obtain another two equations:

$$
\begin{aligned}
& \left(1-q_{1}\right) x \tau_{n}\left(q_{1}^{\alpha} x, y\right) \sigma_{n+1}\left(x, q_{2}^{\beta} y\right)+\left(1-q_{2}\right) y \sigma_{n}\left(q_{1}^{\alpha} x, y\right) \tau_{n+1}\left(x, q_{2}^{\beta} y\right) \\
& -\left(1-q_{1}\right) x\left[1+\left(1-q_{1}\right)\left(1-q_{2}\right) x y\right] \tau_{n}\left(q_{1}^{\alpha} x, q_{2}^{\beta} y\right) \sigma_{n+1} \\
& \quad-\left(1-q_{2}\right) y\left[1+\left(1-q_{1}\right)\left(1-q_{2}\right) x y\right] \tau_{n+1}\left(q_{1}^{\alpha} x, q_{2}^{\beta} y\right) \sigma_{n}=0 .
\end{aligned}
$$

and

$$
\begin{aligned}
& \left(1-q_{1}\right) x \tilde{\sigma}_{n}\left(q_{1}^{\alpha} x, y\right) \tau_{n+1}\left(x, q_{2}^{\beta} y\right)+\left(1-q_{2}\right) y \tau_{n}\left(q_{1}^{\alpha} x, y\right) \tilde{\sigma}_{n+1}\left(x, q_{2}^{\beta} y\right) \\
& -\left(1-q_{1}\right) x\left[1+\left(1-q_{1}\right)\left(1-q_{2}\right) x y\right] \tilde{\sigma}_{n}\left(q_{1}^{\alpha} x, q_{2}^{\beta} y\right) \tau_{n+1} \\
& \quad-\left(1-q_{2}\right) y\left[1+\left(1-q_{1}\right)\left(1-q_{2}\right) x y\right] \tilde{\sigma}_{n+1}\left(q_{1}^{\alpha} x, q_{2}^{\beta} y\right) \tau_{n}=0 .
\end{aligned}
$$

Thus equations (2.19) and (2.31-2.32) represent our Pfaffianized q-difference two-dimensional Toda lattice.

We can write the solutions to this resulting system by choosing the entries in the pfaffians to be expressed in the following form:

$$
\operatorname{pf}(i, j)_{n}(x, y)=\sum_{k=1}^{M}\left[\phi_{k}^{(n+i)}(x, y) \varphi_{k}^{(n+j)}(x, y)-\varphi_{k}^{(n+i)}(x, y) \phi_{k}^{(n+j)}(x, y)\right]
$$


where $\varphi_{k}^{(m)}(x, y)$ and $\phi_{k}^{(m)}(x, y), k=1,2, \cdots, M, M$ being an arbitrary integer, satisfy the equations:

$$
\begin{array}{ll}
\delta_{q_{1}^{\alpha}, x} \phi_{k}^{(m)}(x, y)=-\phi_{k}^{(m+1)}(x, y), & \delta_{q_{2}^{\beta}, y} \phi_{k}^{(m)}(x, y)=\phi_{k}^{(m-1)}(x, y), \\
\delta_{q_{1}^{\alpha}, x} \varphi_{k}^{(m)}(x, y)=-\varphi_{k}^{(m+1)}(x, y), & \delta_{q_{2}^{\beta}, y} \varphi_{k}^{(m)}(x, y)=\varphi_{k}^{(m-1)}(x, y) .
\end{array}
$$

A particular solution to the above equations (2.34-2.35) is obtained by taking $\varphi_{k}^{(m)}(x, y)$ and $\phi_{k}^{(m)}(x, y), k=1,2, \cdots, M$ as following "q-exponential type" functions:

$$
\begin{aligned}
\varphi_{k}^{(m)}(x, y)= & \alpha_{k}\left(-p_{k}\right)^{m} \exp _{q_{1}^{\alpha}}\left(p_{k} x\right) \exp _{q_{2}^{\beta}}\left(-p_{k}^{-1} y\right) \\
& +\tilde{\alpha}_{k}\left(-\tilde{p}_{k}\right)^{m} \exp _{q_{1}^{\alpha}}\left(\tilde{p}_{k} x\right) \exp _{q_{2}^{\beta}}\left(-\tilde{p}_{k}^{-1} y\right), \\
\varphi_{k}^{(m)}(x, y)= & \beta_{k}\left(-s_{k}\right)^{m} \exp _{q_{1}^{\alpha}}\left(s_{k} x\right) \exp _{q_{2}^{\beta}}\left(-s_{k}^{-1} y\right) \\
& +\tilde{\beta}_{k}\left(-\tilde{s}_{k}\right)^{m} \exp _{q_{1}^{\alpha}}\left(\tilde{s}_{k} x\right) \exp _{q_{2}^{\beta}}\left(-\tilde{s}_{k}^{-1} y\right),
\end{aligned}
$$

where $\alpha_{k}, p_{k}, \tilde{\alpha}_{k}, \tilde{p}_{k}, \beta_{k}, s_{k}, \tilde{\beta}_{k}, \tilde{s}_{k}$ are constants, and $\exp _{q^{\alpha}}(x)$ is the q-exponential function defined in the following way [9]:

$$
\begin{aligned}
& \exp _{q^{\alpha}}(x)=\sum_{j=0}^{\infty} \frac{x^{j}}{[j] !}, \quad[j]=\frac{1-\left(q^{\alpha}\right)^{j}}{1-q}, \\
& {[j] !=\left(\frac{1-q^{\alpha}}{1-q}\right)\left(\frac{1-\left(q^{\alpha}\right)^{2}}{1-q}\right) \cdots\left(\frac{1-\left(q^{\alpha}\right)^{j}}{1-q}\right) .}
\end{aligned}
$$

\section{Conclusion and discussion}

We have presented a pfaffianized system of the q-difference version for the two-dimensional Toda lattice equation. This system contains three unknown functions. In fact, q-difference version for the two-dimensional Toda lattice equation can be considered as a reduction of this larger system. This pfaffianized system exhibits solutions expressed in terms of q-exponential functions. Based on the success of pfaffianization in classical soliton equations, we believe that the method of pfaffianization may be further generalized to some other cases, say the supersymmetric case. We hope that this pfaffianized q-difference twodimensional Toda lattice equation (2.19) and (2.31-2.32) will find physical applications.

In the restricted case $q_{1}=q_{2}=q, \alpha=\beta=2$, the q-difference two-dimensional Toda lattice equation( 2.1) or (2.2) is reduced to a q-difference version of the cylindrical Toda lattice equation [7]. It would be of interest to study further if this restriction in the pfaffianized case give a pfaffianized version of a q-difference cylindrical Toda lattice equation.

Acknowledgments. The authors would like to express their sincere thanks to the referee for helpful remarks. This work was partially supported by the National Natural Science Foundation of China (Grant no. 10471139), CAS President grant, the knowledge innovation program of the Institute of Computational Math., AMSS and Hong Kong RGC Grant No. HKBU2016/03P. 


\section{References}

[1] Hirota R and Ohta Y, J. Phys. Soc. Japan 60 (1991), 798.

[2] Gilson C R and Nimmo J J C, Theoret. Math. Phys. 128 (2001), no. 1, 870-882.

[3] Ohta Y, Nimmo J J C, and Gilson C R, Glasg. Math. J. 43A (2001), 99-108.

[4] Jun-Xiao Zhao, Chun-Xia Li, and Xing-Biao Hu ,J. Phys. Soc. Japan 73 (2004), 1159.

[5] Hu X-B, Zhao J-X, and Tam H-W, Pfaffianization of the two-dimensional Toda lattice, J. Math. Anal. Appl., 296 (2004), 256-261.

[6] Gilson C R, Nimmo J J C, and Tsujimoto S, Pfaffianization of the discrete KP equation, $J$. Phys. A 34 (2001), no. 48, 10569-10575.

[7] Kajiwara K and Satsuma J, J. Phys. Soc. Japan 60 (1991), 3986-3989.

[8] Hirota R, Direct Methods in Soliton Theory (in Japanese), (Iwanami) 1992.

[9] Kac V and Cheung P, Quantum Calculus, Springer-Verlag, New York, 2002. 